\title{
Author Correction: Continental scale structuring of forest and soil diversity via functional traits
}

Vanessa Buzzard (D), Sean T. Michaletz (D), Ye Deng (D), Zhili He, Daliang Ning (D), Lina Shen, Qichao Tu, Joy D. Van Nostrand (D), James W. Voordeckers, Jianjun Wang (D), Michael D. Weiser (D), Michael Kaspari, Robert B. Waide, Jizhong Zhou (iD and Brian J. Enquist (D)

Correction to: Nature Ecology \& Evolution https://doi.org/10.1038/s41559-019-0954-7 (2019), published online 19 August 2019.

In the version of this Article originally published there were some errors in the data in Table 1 . For model 5 the $P$ value should have been $2.18 \times 10^{-7}$ not $2.19 \times 10^{-7}$; for model 8 it should have been 0 not $3.0 \times 10^{-4}$; for model 14 it should have been $1 \times 10^{-3}$ not $8.0 \times 10^{-4}$. The $r^{2}$ value for model 7 should have been 0.14 not 0.15 . The AICc value for model 12 should have been 21 not 22 . Further, a technical error led to some values not appearing as bold: for model 4, 0.10 on the bottom arrow; for model 5, 0.15 on the top arrow; for model 13 , the AICc value 7 . All these errors have now been amended.

Published online: 3 October 2019

https://doi.org/10.1038/s41559-019-1014-Z 